Doug Geisler, Eva K. Grebel, and Dante Minniti, eds.

\title{
Mass Segregation in the Young SMC Cluster NGC 330
}

\author{
Marco Sirianni \\ Johns Hopkins University, $3400 \mathrm{~N}$ Charles St, Baltimore MD 21218, \\ USA
}

Antonella Nota, Guido De Marchi

STScI/ESA, 3700 San Martin Drive, Baltimore MD 21218, USA

Claus Leitherer \& Mark Clampin

STScI, 3700 San Martin Drive, Baltimore MD 21218, USA

\begin{abstract}
We present a new study of the low end of the stellar IMF of NGC 330, the richest young star cluster in the Small Magellanic Cloud (SMC). Using deep broadband images taken with the HST/WFPC2 we have derived the cluster's luminosity function and constructed the initial mass function (IMF) in the mass range $1-7 \mathrm{M}_{\odot}$. We have investigated the IMF as a function of the radial distance from the cluster center. We find that, after correction for background contamination, the IMF is fairly homogeneous with a slope slightly steeper than Salpeter's in the central regions of the cluster $\left(<40^{\prime \prime}\right)$ but becomes increasingly steeper with distance, indicating a preponderance of massive stars in the core of the cluster. NGC 330 is one of the first clusters for which evidence of mass segregation is directly found.
\end{abstract}

\section{Introduction}

We present the first results of a systematic study with the HST of a number of young clusters in the LMC and in the SMC, at different conditions of stellar density, age and metallicity. The objective is to investigate the low end of the stellar IMF and to understand whether at small masses the IMF is constrained by local conditions. NGC 330, one of the brightest young clusters in the SMC, has been the first cluster to be studied.

NGC 330 is young (age $<50 \mathrm{Myr}$ ) and very populous. Initial indications of a very peculiar abundance (lower than a factor of 5 with respect to the SMC field young population) have been recently revised by Gonzalez \& Wallerstein (1999), and Hill (1999). Hill (1999) find $[\mathrm{Fe} / \mathrm{H}]=-0.82 \pm 0.11$ dex, which argues in favor of a formation for the cluster from gas which has experienced the same chemical history of the SMC, and strengthens the suitability of NGC 330 for our comparative study. 


\section{Observations}

Multiple images of NGC 330 were obtained in September 1999 using the WFPC2 on board the HST. Several exposures were taken through the filters F555W and F814W. For each filter, a dithered pair of two 350 s exposures was obtained, in addition to a single short (10 s) exposure to image the brightest cluster members. We detect, for the first time, stars down to a limiting magnitude of $m_{F 555 W}=$ 24.9 mag. At the distance of the SMC, assumed to be $59 \mathrm{Kpc}$ (van den Bergh 1999), this corresponds to stellar masses as low as $\sim 0.8 \mathrm{M}_{\odot}$.

PSF fitting photometry was performed, with a conservative threshold set to discard all stars with an associated error larger than 0.2 mag in both filters. We found 3050 stars in the PC and 12674 stars in the WFs.

\section{Color-Magnitude Diagram and Luminosity Function}

We have constructed a color-magnitude diagram (CMD) where $\mathrm{m}_{\mathrm{F} 555 \mathrm{~W}}$ is plotted as a function of the $\mathrm{m}_{\mathrm{F} 555 \mathrm{~W}}-\mathrm{m}_{\mathrm{F} 814 \mathrm{~W}}$ color for all the stars with photometric errors smaller than $\sim 0.2 \mathrm{mag}$ in the combined frames (Figure 1).

In order to determine the cluster age we used isochrones from Lejeune et al. (2001). Isochrone fitting has been restricted to the CMD of the innermost cluster regions (i.e., the PC field, or $r<20^{\prime \prime}$ ), where the effects of background/foreground contamination are less severe. We have translated absolute magnitudes into observed ones assuming a distance modulus $(m-M)_{V}=18.85$ mag and reddening $A_{V}=2.72 E_{B-V}$ mag, $E(B-V)=0.06 \mathrm{mag}$ as appropriate for NGC 330 (Bouchet et al. 1985; Gonzalez et al. 1999). Comparison with the isochrones suggests an age of $30 \mathrm{Myr}$, in excellent agreement with the $\log ($ age $)=7.5$ determined by Keller et al. (2000).

The stellar density is very high in this cluster, and therefore we can study the variations of the luminosity function (LF) with radial distance. We have studied the LF in fourteen annuli between $0^{\prime \prime}$ and $120^{\prime \prime}$ from the center. The annuli are spaced by $5^{\prime \prime}$ between $0-25^{\prime \prime}$, and by $10^{\prime \prime}$ from a distance of $25^{\prime \prime}$ outward. The LF for each annulus has been corrected for field star contamination and for photometric incompleteness.

\section{The Initial Mass Function of NGC 330: Evidence For Mass Seg- regation}

We then converted the LF into a IMF (in the range $1-7 \mathrm{M}_{\odot}$ ) using the appropriate mass-luminosity relation. Although we use the term IMF, we are actually discussing the present-day mass function (MF). At an age of $\sim 30 \mathrm{Myr}$, NGC 330 is young enough that its present day MF should reflect the cluster's IMF, at least for stars that are still on the MS (i.e., for objects less massive than $\sim 8 \mathrm{M}_{\odot}$ ) and are unaffected by dynamical evolution.

We show in Figure 2 the MFs derived for the fourteen annuli. The most remarkable feature of the variation of the MF slope in the different annuli is the pronounced increase of the slope with distance from the cluster center. This trend confirms the marked steepening also observed in the LFs. A careful inspection of the LF in each annulus reveals that the steepening of the MF with 
distance is due to the paucity of massive stars in the periphery rather than to the excess of low mass objects. After correction for background contamination, the slope of the MFs is consistent with a Salpeter's slope out to $40^{\prime \prime}$ from the cluster center, but it becomes considerably steeper in the outer regions.

The observed mass segregation (i.e., the preponderance of massive stars in the core) can be understood as being the result of two mechanisms: internal dynamical evolution, and local variations in star formation. For NGC 330 the half-mass relaxation time is about an order of magnitude longer than the cluster's age, namely $\sim 5 \cdot 10^{8}$ yr (Kontizas et al. 1984) to be compared with an estimated cluster age of $\sim 3 \cdot 10^{7} \mathrm{yr}$. Although it is possible that dynamical evolution has played some role in reshaping the MF in the innermost cluster regions, where the density is high and the relaxation time is short, it is, nevertheless, quite unlikely that internal dynamical evolution might have had enough time to act on the stars throughout the whole cluster and produce the effects that we see in Figure 2, particularly the shortage of massive stars in the outer regions.

This result suggests that the mass distribution at birth, namely the IMF, is a sensitive function of the location in which stars form. The extension of this study to all the other clusters in our sample will allow us to identify any possible correlation between the IMF variations and local conditions.

\section{References}

Bouchet, P., Lequeux, J., Maurice, E., Prevot, L. Prevot-Burnichon, M. L. 1985, A\&A, 149, 330

Gonzalez, G. \& Wallerstein, G. 1999, AJ, 117, 2286

Hill, V. 1999, A\&A, 345, 43

Keller, S.C., Bessel, M.S. \& Da Costa, G.S. 2000, ApJ, 119, 1748

Kontizas, M. 1984 A\&A,131,58

Lejeune, T. \& Schaerer, D. 2001, A\&A, 366, 538

van den Bergh, S. 1999, IAU Symposium 190, Y.-H. Chu, N.B. Suntzeff, J.E. Hesser \& D.A. Bohlender eds., (San Francisco: ASP), 569 

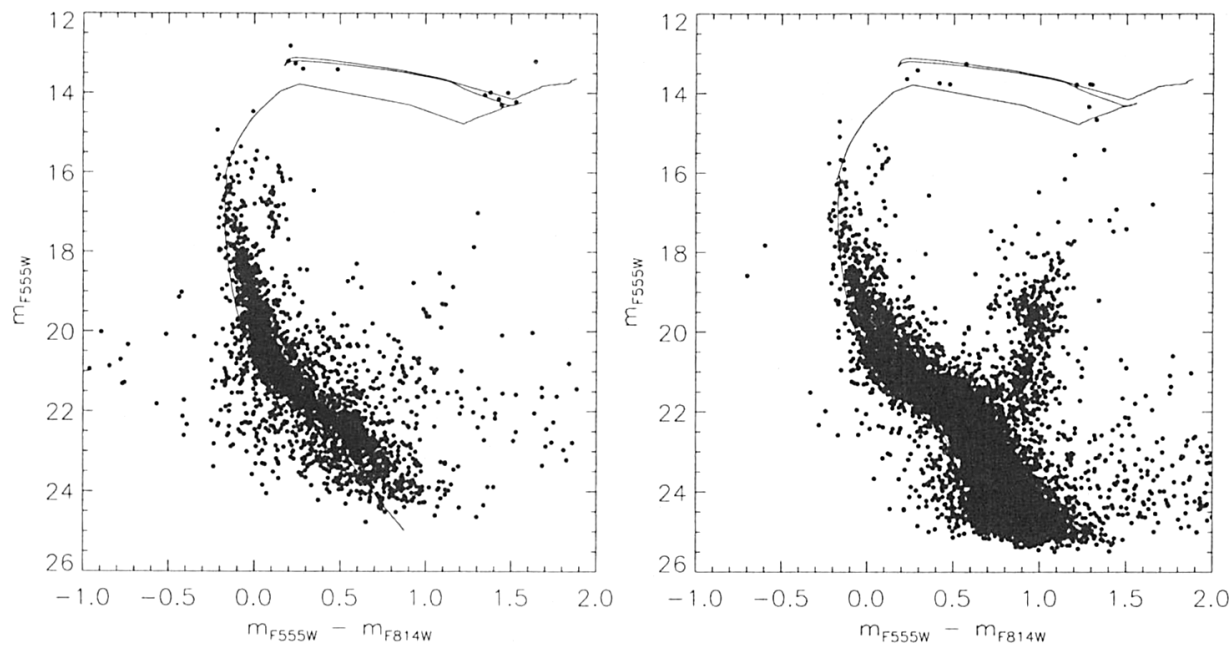

Figure 1. CMD of the inner 25" of NGC330 (left panel) and of the outer regions in the cluster (right panel). The magnitude $m_{F 555 W}$ is plotted as a function of the color $\mathrm{m}_{\mathrm{F} 555 \mathrm{~W}}-\mathrm{m}_{\mathrm{F} 814 \mathrm{~W}}$. The best fitting isochrone $\left(3 \times 10^{7} \mathrm{yr}\right)$ is overplotted for comparison as a solid line.

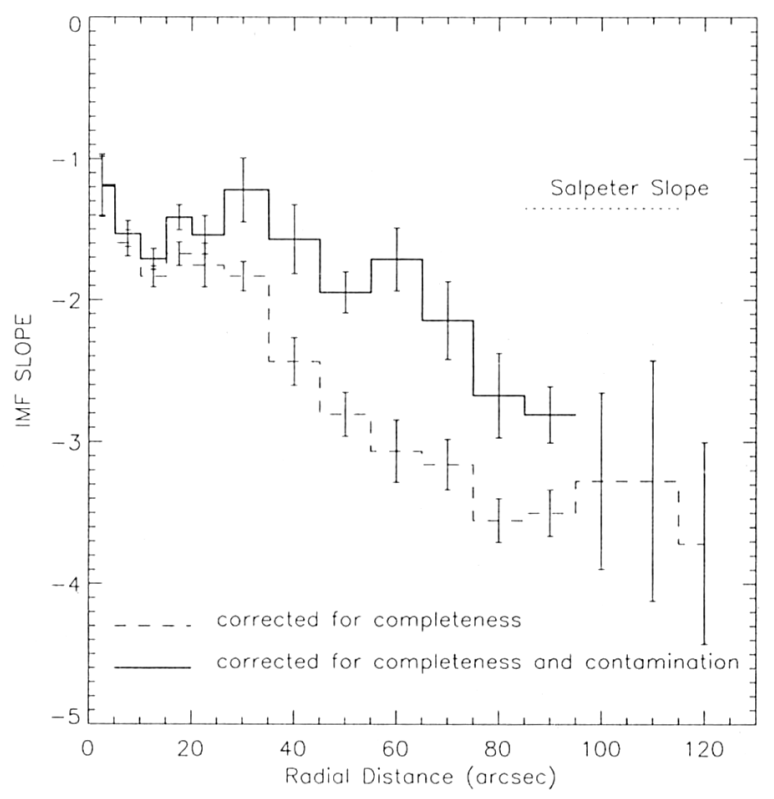

Figure 2. The slopes of the IMFs constructed in equally spaced annuli are here plotted as a function of distance from the cluster center. The dashed lines indicate the values corrected only for completeness, to be compared with the values corrected for completeness and field star contamination (solid line). 